\title{
Rodent ultrasonic vocalizations as biomarkers of future alcohol use: A predictive analytic approach
}

\author{
Nitish Mittal ${ }^{1,2} \cdot$ W. Todd Maddox ${ }^{3} \cdot$ Timothy Schallert ${ }^{2,4} \cdot$ Christine L. Duvauchelle $^{1,2}$
}

Published online: 5 December 2017

(C) Psychonomic Society, Inc. 2017

\begin{abstract}
Excessive alcohol consumption has a vast, negative impact on society. Rodent models have been successful in furthering our understanding of the biological underpinnings that drive alcohol consumption. Rodents emit ultrasonic vocalizations (USVs) that are each composed of several acoustic characteristics (e.g., frequency, duration, bandwidth, power). USVs reflect neurotransmitter activity in the ascending limb of the mesolimbic dopaminergic and cholinergic neurotransmitter systems and serve as noninvasive, real-time biomarkers of dopaminergic and cholinergic neurotransmission in the limbic system. In the present study, we recorded spontaneously emitted USVs from alcohol-naïve Long-Evans (LE) rats and then measured their alcohol intake. We compared the USV acoustic characteristics and alcohol consumption data from these LE rats with previously published data from selectively bred high-alcohol (P and HAD-1) and low-alcohol (NP and LAD-1) drinking lines from studies with the same experimental method. Predictive analytic techniques were applied simultaneously to this combined data set and revealed that (a) USVs emitted by alcohol-naïve rats accurately discriminated among high-alcohol consuming, LE, and low-alcohol consuming rat lines, and (b) future alcohol consumption in these same rat lines was reliably predicted from the USV data collected in an alcohol-naïve state. To our knowledge, this is the first study to show that alcohol consumption is predicted directly from USV profiles of alcohol-naïve rats. Because USV acoustic characteristics are sensitive to underlying neural activity, these findings suggest that baseline differences in mesolimbic cholinergic and dopaminergic tone could determine the propensity for future alcohol consumption in rodents.
\end{abstract}

Keywords Animal models · Alcoholism biomarker · Predictive analytics · Linear discriminant analysis · Long-Evans rats · Selectively bred rats

Excessive alcohol consumption has a vast societal impact with far-reaching consequences for drinkers and the people around them. In the United States, the cost of alcohol misuse from lost

Christine L. Duvauchelle

duvauchelle@mail.utexas.edu

1 College of Pharmacy, Division of Pharmacology and Toxicology, The University of Texas at Austin, 2409 University Avenue, Stop A1915, Austin, TX 78712, USA

2 Waggoner Center for Alcohol and Addiction Research, The University of Texas at Austin, 2500 Speedway, Stop A4800, Austin, TX 78712, USA

3 Cognitive Design and Statistical Consulting, Austin, TX 78746, USA

4 Department of Psychology, Behavioral Neuroscience Division, The University of Texas at Austin, 108 E. Dean Keeton, Stop A8000, Austin, TX 78712, USA workplace productivity, health-care expenses, criminal-justice expense, and motor-vehicle crashes was estimated to be $\$ 249.0$ billion (Sacks, Gonzales, Bouchery, Tomedi, \& Brewer, 2015). Due to the vast cost associated with alcohol abuse, a variety of approaches have been developed to identify the biological underpinnings that drive alcohol consumption.

One such approach in preclinical research is the development of selectively bred rat lines that voluntarily demonstrate excessive alcohol consumption (Murphy et al., 2002). Examples of such lines include alcohol preferring (P) rats and high-alcohol-drinking (HAD-1) rats, as well as their counterparts the alcohol nonpreferring (NP) rats and low-alcoholdrinking (LAD-1) rats. These lines are widely used by laboratories around the world and fit many of the criteria for an animal model of alcohol use disorders (AUDs; Bell et al., 2016; Rodd, Bell, Sable, Murphy, \& McBride, 2004). Research on these lines has led to the identification of therapeutic targets that are also effective in nonselective outbred 
strains, such as the Long-Evans and Wistar rats (Bell et al., 2016; Bell et al., 2012; McBride, Rodd, Bell, Lumeng, \& Li, 2014).

Converging lines of evidence from the selectively bred rat lines and human literature suggest that genetic variation in dopaminergic and cholinergic neurotransmitter systems is associated with alcohol use and abuse (Enoch \& Goldman, 2001; McBride, Kimpel, McClintick, Ding, Hyytia, et al., 2013b; Morzorati \& Marunde, 2006; Stewart \& Li, 1997; Tawa, Hall, \& Lohoff, 2016). One approach to studying the role of cholinergic and dopaminergic neurotransmission in AUDs is to use biomarkers that are associated with activity in these neurotransmitter systems.

Ultrasonic vocalizations (USVs) are an established, noninvasive, real-time, functional biomarker of dopaminergic and cholinergic transmission in the rodent limbic system (Brudzynski, 2015). USVs are separated into two common subtypes: $50-55-\mathrm{kHz}$ frequency modulated (FM) USVs, which are emitted in response to rewarding stimuli and reflect positive affective states (Ahrens, Ma, Maier, Duvauchelle, \& Schallert, 2009; Avvisati et al., 2016; Brenes et al., 2016; Buck, Vendruscolo, Koob, \& George, 2014; Burgdorf \& Panksepp, 2006; Ciucci et al., 2009; Maier, Abdalla, Ahrens, Schallert, \& Duvauchelle, 2012; Popik et al., 2014; RoccaroWaldmeyer, Babalian, Müller, \& Celio, 2016; Williams \& Undieh, 2016) and 22-28-kHz USVs, which are emitted in response to aversive stimuli and reflect negative affect (Bardin et al., 2010; Berger, Williams, McGinnis, \& Walker, 2013; Brudzynski, Bihari, Ociepa, \& Fu, 1993; Chen, Li, Li, \& Kirouac, 2012; Furlanetti, Coenen, Aranda, \& Döbrössy, 2015; Lindquist, Jarrard, \& Brown, 2004; Parsana, Moran, \& Brown, 2012; Wang, Liang, Burgdorf, Wess, \& Yeomans, 2008).

As real-time, functional biomarkers of activity in the mesolimbic dopaminergic and cholinergic neurotransmitter systems (Brudzynski, 2015; Wöhr \& Schwarting, 2013), USVs can serve as important tools for understanding the role of these systems in AUDs as well as a number of other neurological disorders (Basken, Connor, \& Ciucci, 2012; Brenes et al., 2016; Ciucci et al., 2009; Machold, 2013; Pan et al., 2014; Scattoni, Crawley, \& Ricceri, 2009; Steele, Whitehouse, Aday, \& Prus, 2017). However, due to the time-intensive and labor-intensive nature of USV analyses, this methodology is vastly underutilized. In fact, nearly all published USV studies report only short durations of USV activity (e.g., less than 30 minutes) in counts alone (Armstrong et al., 2001; Brudzynski, 2015; Burgdorf, Wood, Kroes, Moskal, \& Panksepp, 2007; Johnson, Grant, Schallert, \& Ciucci, 2015; Kaniuga et al., 2016; Portfors, 2007; Scattoni et al., 2009) but do not provide a full USV profile that could be revealed by repeated, long-duration assessment periods. Our development of WAAVES, a highly accurate algorithm that automates the analysis of USVs (Reno \& Duvauchelle, 2014;
Reno, Marker, Cormack, Schallert, \& Duvauchelle, 2013) has allowed us to analyze data from experiments with relatively long time lines, something that was previously not feasible using manual techniques. In addition, WAAVES also allows an examination of each USV in much greater detail. Specifically, in addition to counting the number of USVs, the WAAVES analyses quantify several acoustic characteristics associated with each USV. These characteristics include the USV mean frequency in kilohertz, duration in milliseconds, bandwidth in kilohertz, and relative power in decibels. The result is a rich, multidimensional representation of each unique USV. These characteristics can be combined into large data sets for analyses using advanced statistical models and predictive analytics to examine the intricate relationships between rat USV characteristics and behavioral phenotypes, such as alcohol seeking and consumption. This is the approach taken in some of our recent work (Mittal et al., 2017; Reno et al., 2017) and the current report.

\section{Overview of the present study}

The overriding aim of the present work is to provide the first empirical test of the hypothesis that rodent ultrasonic vocalizations emitted by alcohol-naïve rats can be used to predict their future alcohol consumption. To test this hypothesis, we conducted a new study using Long-Evans rats and merged these results with our published data obtained from selectively bred high-alcohol ( $\mathrm{P}$ and HAD-1) and low-alcohol (NP and LAD-1) drinking lines. The result is an integrative analysis of USV data collected from five different rat lines that highlight a novel and powerful role of USVs as direct predictors of excessive alcohol consumption and biomarkers of neurotransmission in these rat lines.

Instead of focusing on a single strain of rat, in the present study, we take the more challenging, but more broadly informative approach of testing our hypothesis across a number of rat types including selectively bred lines and a classically bred strain. With this goal in mind, we conducted a new experiment in Long Evans (LE) rats using the same procedures used in two previous studies in our lab, one conducted using $\mathrm{P}$ versus NP rats (Reno et al., 2017) and the other using HAD-1 versus LAD-1 rats (Mittal et al., 2017).

The rest of this manuscript is organized as follows. In the next section, we introduce the new study using Long-Evans rats. This is followed by a description of the predictive analytic approach and results from the combined sample that includes the new Long-Evans data and the P/NP (Reno et al., 2017) and HAD-1/LAD-1 (Mittal et al., 2017) studies. We conclude with a summary and integration of these results with the existing literature. 


\section{Method}

\section{Animals}

Sixteen male Long-Evans rats were ordered from Harlan (Harlan Laboratories, Indianapolis, Indiana) at approximately 4 weeks of age. The average weight of the rats upon arrival was $\sim 180$ grams. Rats were handled 20 minutes per day, 5 days per week for 3 weeks to habituate them to the experimenters. The animals were housed in a 12-hour reverse-light cycle with lights out from 10:00 a.m. to 10:00 p.m. All USV recording sessions and the subsequent $\mathrm{EtOH}$ availability sessions began at the beginning of the dark cycle at 10:00 a.m. Rats had ad libitum access to food and water throughout all experiments. Data from the previously published studies consists of six HAD-1, six LAD-1 (Mittal et al., 2017), 10 P, and four NP rats (Reno et al., 2017). All experiments were conducted in accordance with Institutional Animal Care and Use Committee guidelines.

\section{Ultrasonic vocalization recordings}

USVs were recorded from alcohol-naïve rats in 4-hour sessions for 3 days/week (e.g., Monday, Wednesday, Friday) for 2 weeks. CM16 microphones were used with an UltraSound Gate interface (Avisoft Bioacoustics) to record USVs at a 250$\mathrm{kHz}$ sampling rate and a 16-bit resolution. The recording sessions started at the beginning of the dark cycle. The animals were weighed and transported to the recording room where they were transferred into recording cages, which were identical to the home cages but used only for the recording sessions. Since novelty or conspecific scents could affect rodent behaviors (Wöhr, Houx, Schwarting, \& Spruijt, 2008), each animal was assigned its own recording cage. The animals were given access to food and water in the cage. Based on rat and chamber size, we approximate the distance between the animal's head and the centered microphone to range from $5 \mathrm{~cm}$ to $28.4 \mathrm{~cm}$. Following the recording sessions, the animals were transported back to the vivarium.

\section{Analysis of USVs}

USV recordings were analyzed using the WAAVES program developed in our laboratory (Reno \& Duvauchelle, 2014; Reno et al., 2013). This program reads USV containing audio files and produces a frequency spectrogram, which is subsequently analyzed using MATLAB's Image Processing Toolbox
(MathWorks, Inc., Natick, MA). The program utilizes a variety of filters to separate USV calls from background noise, and several measurements of interest are quantified and extracted from each call. For 50-55-kHz FM USVs, WAAVES identifies sound objects with a minimum duration of $5 \mathrm{~ms}$ occurring in a range of 30 to $120 \mathrm{kHz}$. An intercall interval of $10 \mathrm{~ms}$ was used to discriminate between individual calls and avoid counting call fragments as separate calls. FM USVs were defined as calls that varied more than $5 \mathrm{kHz}$ over the entire duration of the call, and $22-28-\mathrm{kHz}$ calls were identified as sound objects occurring in a frequency range of 20 to $30 \mathrm{kHz}$ with a minimum duration of $200 \mathrm{~ms}$. An intercall interval of $100 \mathrm{~ms}$ was used to separate individual calls. Once the calls are identified, several measurements of interest are extracted from each USV call and stored for subsequent analysis. In the present study, the mean frequency, duration, bandwidth, and power (e.g., in decibel measurements relative to sound pressure at human detection levels) for $50-55-\mathrm{kHz}$ FM and 22-28-kHz USV calls were extracted for statistical analysis.

\section{Validation process for WAAVES automation}

Validation of WAAVES-generated USV data requires correspondence with human-derived analyses. Experimenters manually analyzed subsets of USV data recorded during the experiment to compare human assessment with WAAVES output. A subset of 10-min USV files recorded from LE rats (2228-kHz calls: 40 files; $50-55-\mathrm{kHz}$ calls: 40 files) were used for manual validation. The total number of calls identified via manual analysis was correlated with the total number of calls identified by the automated WAAVES program. The correlation coefficients are reported in the results.

\section{Ethanol availability sessions}

Following the USV recording rats were given access to 24hour chronic intermittent ethanol sessions 3 days/week for 4 weeks as previously described (Mittal et al., 2017; Reno et al., 2017). The sessions began at the beginning of the dark cycle. The rats were weighed at the beginning of each session and received three bottles containing either water, $15 \% \mathrm{EtOH}$, or $30 \% \mathrm{EtOH}$. The bottles were removed 24 hours later and weighed to measure ethanol or water consumption. Any potential loss of fluids due to spillage were insignificant, and therefore not accounted for in the present study. The dose of $\mathrm{EtOH}$ in $\mathrm{g} / \mathrm{kg}$ was measured using the following formula:

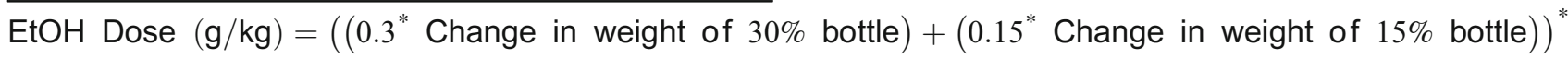

$$
0.8^{*}\left(1 /\left(0.001^{*} \text { Weight of rat in grams }\right)\right) \text {. }
$$




\section{Advanced statistical methods for testing the USV-alcohol consumption relationship}

Traditional USV studies have focused primarily on differences in total call counts in the $22-28-\mathrm{kHz}$ and $50-55-\mathrm{kHz}$ FM categories, across experimental groups. This approach has proven to be a reliable index of underlying neurotransmission and is sensitive to experimental and pharmacological manipulations (Brudzynski, 2013; Ciucci et al., 2009; Knutson, Burgdorf, \& Panksepp, 2002; Pan et al., 2014). Using this approach our lab has shown that USVs are responsive to a wide range of drugs of abuse and can serve as a useful model for investigating the neural basis of drug reward and the development of drug craving, behavioral sensitization and drug reward tolerance (Ahrens et al., 2009; Ahrens et al., 2013; Ma, Maier, Ahrens, Schallert, \& Duvauchelle, 2010; Maier et al., 2012; Reno et al., 2015; Thakore et al., 2016). Many of these studies, including those from our lab, have used standard parametric statistical approaches (e.g., repeated-measures or twoway ANOVAs) to analyze the USV count data. However, these methods are not sufficiently powerful to analyze the USV acoustic characteristic data captured with WAAVES.

Predictive analytic approaches, such as linear discriminant analysis (LDA), are required when the goal is to determine whether a linear combination of the USV acoustic characteristics can successfully discriminate between rat lines (e.g., $\mathrm{P}$ vs. NP or HAD-1 vs. LAD-1 rats). Using LDA on the USV data produces a linear equation that takes the following form:

$$
\begin{aligned}
\text { LDA score }= & \beta_{\text {Mean Frequency }}{ }^{*} \text { Mean Frequency } \\
& +\beta_{\text {Duration }}{ }^{*} \text { Duration } \\
& +\beta_{\text {Bandwidth }}{ }^{*} \text { Bandwidth } \\
& +\beta_{\text {Power }}{ }^{*} \text { Power. }
\end{aligned}
$$

This method computes the $\beta$ coefficients that can be used to combine the data from all four acoustic characteristics into a singular LDA score that maximizes the percent of USV calls classified into the appropriate experimental classes (e.g., P vs. NP or HAD-1 vs. LAD-1 rats). For example, if we have a dataset consisting of 22-28-kHz calls made by $\mathrm{P}$ and NP rats, then the LDA method will produce $\beta$ coefficients that maximize the difference between the LDA scores of the $\mathrm{P}$ and the NP rats respectively, such that we can take the average LDA scores of each group and construct a decision threshold halfway between the two averages to make predictions about unclassified data. That is, if the LDA score (calculated using the equation above) of an unknown animal falls below the decision threshold, then we predict that the rat is a $\mathrm{P}$ rat and if it is above the threshold then we predict that the rat is an NP rat (or vice-versa). An additional advantage of this method is that we are able to reduce the four USV characteristics into a onedimensional LDA score, which can be easily plotted. The LDA method can be implemented using the freely available R package "MASS" (Venables \& Ripley, 2002).

\section{Results}

\section{Using LDA to accurately separate selectively-bred high-drinking and low-drinking rat lines}

Our lab has successfully used linear discriminant analysis to classify HAD-1 and LAD-1 rats with $100 \%$ accuracy solely on the basis of the $50-55-\mathrm{kHz}$ FM USV data (Mittal et al., 2017). Similarly, we have also been able to use $22-28-\mathrm{kHz}$ USV data to discriminate alcohol-naïve HAD-1 rats from LAD-1 rats with a classification accuracy of $81.96 \%$ (Mittal et al., 2017) and alcohol-naïve P rats from NP rats with $100 \%$ accuracy (Reno et al., 2017). Our work on the differences in USV characteristics between the HAD-1/LAD-1 and the P/NP lines suggests that traits associated with alcohol consumption in these lines may also affect USV production. Consequently, USVs may serve as a potential biomarker for excessive alcohol consumption. However, it is also possible that the differences in USV characteristics between these lines may simply have been a byproduct of genetic drift stemming from potential inbreeding within each selectively-bred line (Crabbe, Phillips, Kosobud, \& Belknap, 1990). The P and NP rats were derived from a closed colony of Wistar rats, while the HAD-1 and LAD-1 rats were developed using the N/Nih heterogenous stock (Bell et al., 2012; Gongwer, Murphy, McBride, Lumeng, \& Li, 1989). Therefore, it is unlikely that the similar differences in USV characteristics observed across these different high and low alcohol consuming lines, derived from distinct breeder stocks, are simply a result of random genetic drift during the selective-breeding process.

\section{Using LDA to predict alcohol consumption in a combined HAD-1, LE, LAD-1 sample}

Long-Evans rats, derived from mating a Wistar female with a wild grey rat (Weisbroth, 1969), are commonly used by many labs to study alcohol use and abuse. Although derived from a different breeder stock, HAD-1 and LAD-1 rats show stratification in alcohol consumption levels. Therefore, we first examined alcohol consumption in the combined sample as shown in Fig. 1a. We saw that while the HAD-1 rats consume high levels of alcohol and the LAD-1 rats consumed low levels of alcohol, the LE rats show moderate levels of consumption ranging from HAD-1 levels to LAD-1 levels (see Fig. 1a). Second, we assessed whether similar stratification could be observed in the USV data amongst the HAD-1, 


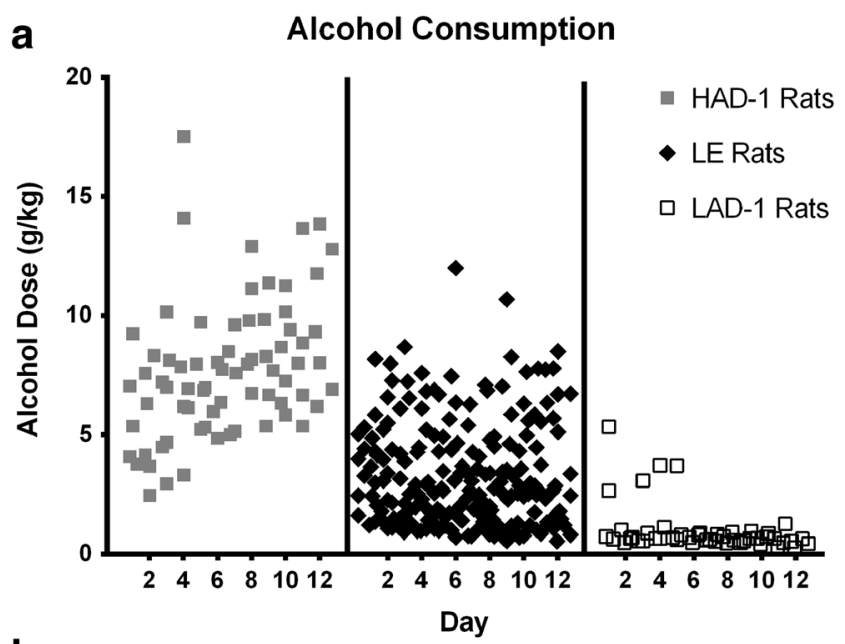

b

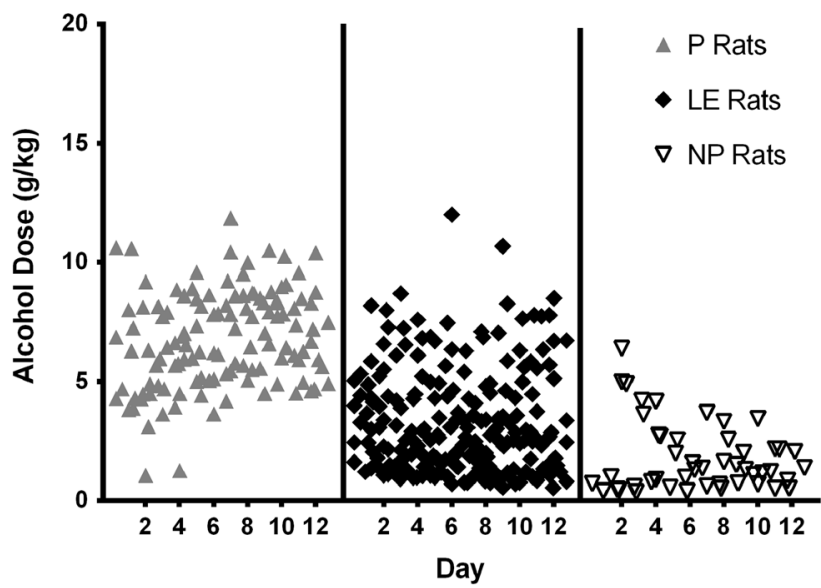

Fig. 1 Daily alcohol consumption of HAD-1-LE-LAD-1 and P-LE-NP rats across 12 drinking sessions. Individual symbols represent individual rats on each of the $12 \mathrm{EtOH}$ session, with a slight offset on the $x$-axis used to minimize overlap between symbols. a Daily alcohol consumption ranged from 2.45 to $17.53 \mathrm{~g} / \mathrm{kg}$ for HAD-1 rats, from 0.55 to $12.00 \mathrm{~g} /$ $\mathrm{kg}$ for LE rats, and from 0.28 to $5.34 \mathrm{~g} / \mathrm{kg}$ for LAD-1 rats. b Daily alcohol consumption ranged from 1.06 to $11.86 \mathrm{~g} / \mathrm{kg}$ for $\mathrm{P}$ rats, from 0.55 to 12.00 $\mathrm{g} / \mathrm{kg}$ for LE rats, and from 0.36 to $6.39 \mathrm{~g} / \mathrm{kg}$ for NP rats

LAD-1, and LE rat lines. All of the USVs emitted during the 2-week period were used for this analysis without any references to recording days. Using LDA, we estimated the linear discriminant scores for the three rat lines. When the LDA method is used to discriminate between two classes of data a single linear equation is produced to generate a decision threshold that separates the two classes. However, when dealing with three classes two decision thresholds are needed. Therefore, the LDA method produced two linear discriminant scores for each of the 50-55-kHz FM (see Fig. 2a) and 22-28kHz USV (see Fig. 2b) categories.

We found that the linear discriminant scores from 50-55kHz FM USVs showed some degree of separation between the HAD- 1 and LE rats (90.91\% separation), and the HAD-1 and LAD-1 rats (100\% separation; see Fig. 2a), though no clear distinction existed between the LDA scores from the LE and LAD-1 rats (54.54\% separation). On the other hand, the LDA scores from the $22-28-\mathrm{kHz}$ USV data showed clear clustering across the HAD-1, LAD-1, and LE rat lines (HAD1 vs. LE separation: $100 \%$; LAD-1 vs. LE separation: 95.45\%; HAD-1 vs. LAD-1 separation: $100 \%$; see Fig. 2b). Because $50-55-\mathrm{kHz}$ FM and 22-28-kHz USV emissions are mediated through the mesolimbic dopaminergic and cholinergic neural pathways (Brudzynski, 2015; Wöhr \& Schwarting, 2013), these results suggest that basal differences in these transmitter systems may exist between the selectively bred $\mathrm{HAD} / \mathrm{LAD}$ and the outbred LE rat lines. However, as no study to date has directly explored these differences across these three rat lines, this hypothesis requires further work for serious consideration.

Linear discriminant scores predict future alcohol consumption Equipped with the linear discriminant scores based on the USV data from alcohol-naive rats and the future alcohol consumption data, we tested the hypothesis that alcohol consumption levels in each rat can be predicted from their USV acoustic characteristics. To test this hypothesis, we generated a multivariate regression model that used the four linear discriminant values described above (i.e., from Fig. 2a-b) to retrospectively predict total alcohol consumption for each rat during the subsequent alcohol availability sessions. Multivariate linear regression modeling strongly supported our hypothesis and revealed that the linear discriminant scores could be used to predict future alcohol consumption in these rat lines (multiple $r^{2}=.7532$ ), $F(4,23)=17.55, p<.0001$. Critically, to ensure that this linear model did not overfit the data, we used the leave-one-out cross-validation method. This is a commonly used validation technique that yields a conservative estimate of the accuracy of the regression model (Kearns \& Ron, 1999). Using this method, for every rat, a model is trained excluding that rat and the model is then used to make predictions on the data from the excluded rat. Thus, the model predicted values are generated on unseen data that are not used in building the model. As shown in Fig. 3a, the model predicted consumption values showed a high degree of correlation with the total alcohol consumption of the HAD-1, LAD-1, and LE rats during the 4-week alcohol access period $\left(r^{2}=.5999\right), F(1,26)=38.99, p<.0001$ (se Fig. 3a). This finding provides the first direct evidence that USV acoustic characteristics can predict future alcohol consumption in alcohol-naïve rats. Importantly, this finding is not focused on a single strain of rats, but rather holds across three strains that are commonly used in alcohol research. The breadth of this finding across strains attests to its generality.

One might argue that by including selectively bred highalcohol and low-alcohol consuming lines in the multivariate regression we increased the likelihood of finding that USVs predict future alcohol consumption. This reasoning is 
HAD-LAD-LE Linear Discriminant Scores

a

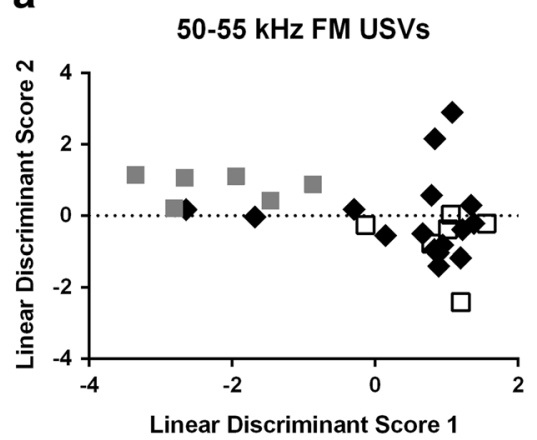

b

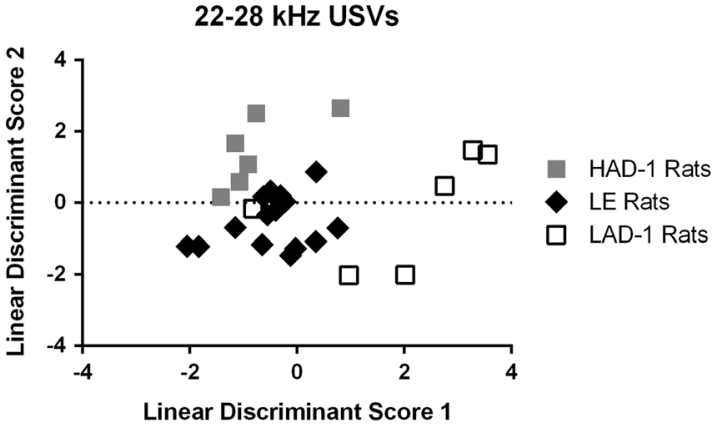

P-NP-LE Linear Discriminant Scores

C

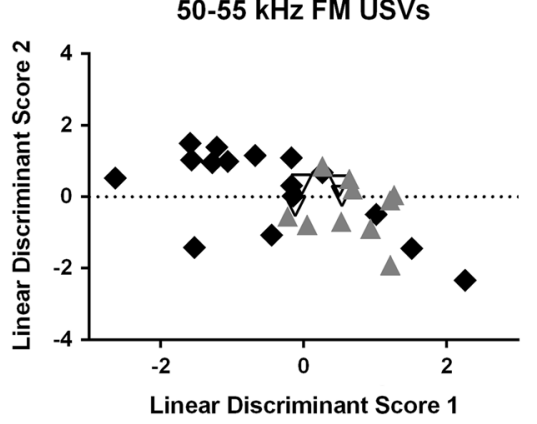

Fig. 2 Maximal separation between HAD-1-LE-LAD-1 and P-LE-NP rats achieved via linear discriminant analysis using $22-28-\mathrm{kHz}$ and 50 $55-\mathrm{kHz}$ FM USV data. a Linear discriminant analysis provided $90.91 \%$ separation accuracy between HAD-1 and LE rats, and 100\% separation accuracy between HAD-1 and LAD-1 rats based on 50-55-kHz FM USV data. No clear separation was observed between LE and LAD-1 rats. b Linear discriminant analysis based on $22-28-\mathrm{kHz}$ USV data showed $100 \%$ separation between HAD-1 and LE rats, $95.45 \%$ separation

problematic because it assumes that USV profiles differ across these lines in a way that is systematically related to alcohol consumption. This is an empirical question that is tested in the current report. Even so, we conducted a secondary analysis focused just on the LE rats. In this LE subsample, we continue to find that alcohol consumption is strongly predicted from the USV profiles, $F(1,14)=12.90, p<.01$.

\section{Using LDA to predict alcohol consumption in a combined $P$, LE, NP sample}

Given the shared ancestry between the LE and the P/NP rat lines, we also sought to investigate potential similarities or differences in USV acoustic characteristics among these three lines. As shown in Fig. 1b, the alcohol consumption of LE rats also ranges from NP rat consumption levels to $\mathrm{P}$ rat consumption levels. Therefore, we also tested whether linear discriminant scores could be used to accurately separate the LE rats from the P and the NP rats. As seen in Fig. 2a, the linear discriminant scores

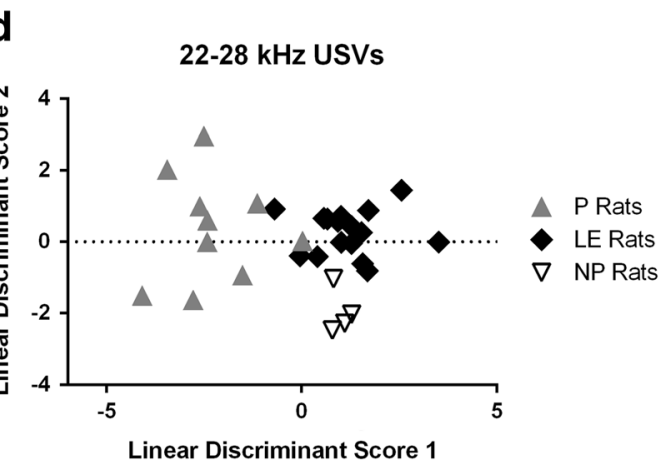

between LAD-1 and LE rats, and 100\% separation accuracy between HAD-1 and LAD-1 rats. c Linear discriminant analysis based on 50 $55-\mathrm{kHz}$ FM USV data did not show any clear separation between the $\mathrm{P}$, Len, and NP rat lines. d Linear discriminant analysis based on $22-28-\mathrm{kHz}$ USV data showed $92.31 \%$ separation between $\mathrm{P}$ and LE rats, $95.00 \%$ separation between NP and LE rats, and 100\% separation accuracy between $\mathrm{P}$ and NP rats

from the 50-55-kHz FM USV data did not show any clear distinction between the three rat lines (P vs. LE separation: $80.77 \%$; NP vs. LE separation: $65.00 \%$; P vs. NP separation $71.43 \%$ ). However, the $22-28-\mathrm{kHz}$ USV associated LDA values showed clear clustering across the P, NP, and LE rats (P vs. LE separation: $92.31 \%$; NP vs. LE separation: $95.00 \%$; P vs. NP separation 100\%; see Fig. $2 \mathrm{~b})$. Since $22-28-\mathrm{kHz}$ USVs are associated with tegmental cholinergic transmission, these results suggest that there may be differences in basal cholinergic activity in the limbic regions across these three lines. No study has directly compared these neurotransmitter systems between $\mathrm{P}$ and LE rats. Together with the HAD/LAD results, these findings highlight a need for future studies to directly explore these systems and compare neurotransmitter activity across these rat lines.

Linear discriminant scores predict future alcohol consumption Next, we tested whether the linear discriminant scores based on the USV data from alcohol-naïve rats could also 
a

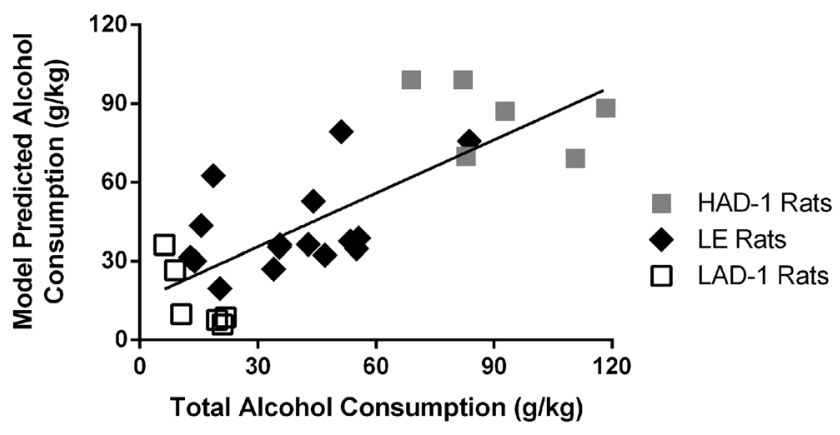

b

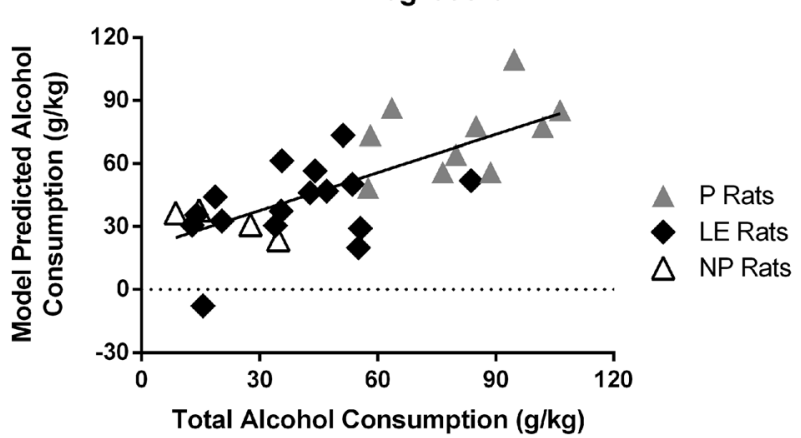

Fig. 3 Comparison of total alcohol consumption and model predicted alcohol consumption of HAD-1-LE-LAD-1 and P-LE-NP rats during 4 weeks of 24-hour alcohol availability sessions. a Multivariate linear regression models developed using linear discriminant scores from HAD-1, LE, and LAD-1 rats showed a high degree of correlation between the model predicted alcohol consumption and the actual consumption of these rats $\left(r^{2}=.5999\right), F(1,26)=38.99, p<.0001$. b Multivariate linear regression models developed using linear discriminant scores from $\mathrm{P}, \mathrm{LE}$, and NP rats also showed a high degree of correlation between the model predicted alcohol consumption and the actual consumption of these rats $\left(r^{2}=.5112\right), F(1,28)=29.29, p<.0001$

predict alcohol consumption in these rats. ${ }^{1}$ Multivariate linear regression models showed that these LDA score could also be used to predict alcohol consumption in these rats (multiple $r^{2}$ $=.6995), F(4,25)=14.55, p<.0001$. The model predicted consumption values, generated using the leave-one-out crossvalidation method described above, showed a significant correlation with the alcohol consumption of the $\mathrm{P}, \mathrm{NP}$, and LE rats $\left(r^{2}=.5112\right), F(1,28)=29.29, p<.0001$. Figure 3 b provides a representation of this relationship using the assigned and

\footnotetext{
${ }^{1}$ The USV data for the P/NP rats used in this study were collected from the control group of the P/NP study (Reno et al., 2017). Thus, these rats did not receive alcohol following the USV recording experiment, making it difficult to assess the ability of USV data to predict future alcohol consumption in these rats. In order to overcome this problem, we used a random sampling method, whereby each of the $\mathrm{P}$ and NP rats used in this study were randomly assigned the alcohol consumption value of a $\mathrm{P}$ or NP rat from the alcohol-experience group of the P/NP study (Reno et al., 2017), and a linear model was generated to predict the alcohol-consumption values of these rats based on the USV data. This process was repeated 10,000 times, and the average prediction values from the 10,000 iterations were used to generate the reported correlation coefficients.
}

predicted alcohol consumption values from one of the 10,000 simulations. These findings complement the HADLAD-LE data described above and provide strong evidence that USV data from selectively bred lines can be generalized to Long-Evans rats. Together, these results provide clear and direct evidence that USV data can be used to predict alcohol consumption in rats.

As with the HAD/LAD analysis, one might argue that by including selectively bred high-alcohol and low-alcohol consuming lines in the multivariate regression, we increased the likelihood of finding that USVs predict future alcohol consumption. To address this potential concern, we conducted a secondary analysis focused just on the LE rats. In this LE subsample, we continue to find that alcohol consumption is strongly predicted from the USV profiles, $F(1,14)=30.77, p$ $<.0001$.

\section{Validation of USV analysis}

A subset of USV files were manually analyzed using Avisoft SASLab Lite (Avisoft Bioacoustics) to validate WAAVES output. WAAVES-automated analysis and manual analysis results were highly correlated for both the $22-28-\mathrm{kHz}(r=$ $.996, p<.0001)$ and $50-55-\mathrm{kHz}$ calls $(r=.970, p<.0001)$.

\section{Discussion}

The present study provided the first known test of the hypothesis that rodent ultrasonic vocalizations obtained while animals are alcohol naïve serve as predictors for future alcohol consumption. Instead of focusing on a single rat strain, we took the more challenging, but more general, approach of testing this hypothesis across five rat lines: P, NP, HAD-1, LAD-1, and Long-Evans. We report the results from a new study with Long-Evans rats that uses the same procedure used in two other studies conducted in our lab: one with P and NP rats (Reno et al., 2017), and the other with HAD-1 and LAD-1 rats (Mittal et al., 2017). In all three studies, we recorded spontaneously emitted USVs while the rats were alcohol naive and followed this with free alcohol access sessions in which alcohol consumption was measured. We combined these three data sets and utilized predictive analytic techniques to test this hypothesis.

Here, we show that differences in USV expression, which are thought to be mediated through mesolimbic dopaminergic and cholinergic neurotransmitter systems, are linked to the propensity for alcohol consumption in selectively bred and nonselectively bred rat lines. We showed that USVs could serve as real-time functional markers of activity in these systems. Two major findings emerged. First, we showed that linear discriminant scores calculated using the USV data could be used to discriminate Long-Evans rats from rat lines 
selectively bred for high or low levels of alcohol consumption. These results point to potential differences in neurotransmission across these five rat lines and highlight the need for direct investigation of these systems. Second, using multivariate linear regression, we found that future alcohol consumption could be predicted from USV profiles obtained while animals were alcohol naïve. Thus, these scores provide information about basal neurotransmission in these rat lines, unencumbered by any potential disruptions from alcohol exposure and highlight a novel and potentially invaluable use of USV data as a predictor of alcohol consumption in these five rat lines.

Both pairs of rat lines tested in this study are known to have basal differences in dopaminergic and cholinergic transmission. For instance, $\mathrm{P}$ and HAD-1 rats have lower tissue levels of DA and its metabolites (DOPAC and HVA) in the nucleus accumbens and the anterior striatum as compared to their NP and LAD-1 counterparts (Gongwer et al., 1989; Murphy, McBride, Lumeng, \& Li, 1982, 1987). This may be due to a potential deficit in the VTA DA projections in these rat lines. Indeed, P rats have reduced DA projections from the VTA to the nucleus accumbens (Zhou, Zhang, Lumeng, \& Li, 1995) as well as lower levels of DA $\mathrm{D}_{2}$ receptor binding sites (McBride, Chernet, Dyr, Lumeng, \& Li, 1993), as compared to the NP rats. In order to compensate for the lower levels of DA, P rats show increased burst firing of VTA DA neurons, though a similar increase was not seen in the HAD rats (Morzorati, 1998; Morzorati \& Marunde, 2006). Alcohol consumption results in reduced $\mathrm{D}_{2}$ auto-receptor function (Engleman, McBride, Li, Lumeng, \& Murphy, 2003; Engleman et al., 2000), and enhanced DA efflux (Thielen et al., 2004; Weiss, Lorang, Bloom, \& Koob, 1993) and reuptake (Sahr, Thielen, Lumeng, Li, \& McBride, 2004).

The potential differences in cholinergic transmission between the selectively bred rat lines remain largely unexplored. However, the few studies that have been conducted suggest that the accumbal cholinergic system may be more active in $\mathrm{P}$ rats than NP rats (Bell et al., 2016). For instance, P rats have lower expression levels of striatal nicotinic acetylcholine receptor than NP rats (Tizabi, Getachew, Davila-Garcia, \& Taylor, 2001). Studies have also identified higher expression levels of genes encoding choline acetyltransferase, muscarininc acetylcholine receptor 3 , and channels responsible for the uptake and vesicular transport of acetylcholine during synthesis in the nucleus accumbens shell of $\mathrm{P}$ rats as compared to the NP rats (McBride, Kimpel, McClintick, Ding, Hauser, et al., 2013a; McBride, Kimpel, McClintick, Ding, Hyytia, et al., 2013b). Furthermore, administration of nicotinic antagonists or desensitizing agents can reduce alcohol consumption in P rats (Rezvani et al., 2010; Srisontiyakul, Kastman, Krstew, Govitrapong, \& Lawrence, 2016). Although we did not directly explore these systems in our study, these studies provide support to the notion that the
USV differences across these lines are a reflection of underlying differences in these neurotransmitter systems.

Although USV recordings are noninvasive and USV recording experiments are relatively easy to set up, USVs have remained relatively underutilized as important preclinical tools for the investigation of neural substrates underlying alcohol and drug abuse, primarily due to the manually intensive nature of the USV analysis process. The development of WAAVES (Reno \& Duvauchelle, 2014; Reno et al., 2013), and other automated USV detection methods (Barker, Herrera, \& West, 2014; Barker \& Johnson, 2017) can help these problems and should encourage more labs to integrate USV recordings to their respective batteries of behavioral assays. Widespread use of USVs can unlock the true potential of this behavioral metric as a noninvasive correlate of underlying neural activity.

\section{Conclusion}

This present study provides the first known test of the hypothesis that rodent ultrasonic vocalizations obtained while animals are alcohol naïve serve as a biomarker for and thus predict future alcohol consumption. We find support for this hypothesis in a combined sample of five rat types commonly used in alcohol research. These results highlight a novel role of USVs as biomarkers of neurotransmission and the propensity for high levels of alcohol consumption in rodents.

Acknowledgements Research support: Gates Millennium Scholarship Program (NM), AA013517 (CLD), University of Texas Waggoner Center for Alcohol and Addiction Research. We would like to thank Dr. John G. Hixon for lively discussion and advice on the statistical methods employed in this study. We would also like to thank Anuska Martinez, Eunjoon Park, Hannah Trinh, Sanaa Prasla, and Sebrina Yan for their contributions with data collection and analysis.

\section{References}

Ahrens, A. M., Ma, S. T., Maier, E. Y., Duvauchelle, C. L., \& Schallert, T. (2009). Repeated intravenous amphetamine exposure: Rapid and persistent sensitization of $50-\mathrm{kHz}$ ultrasonic trill calls in rats. Behavioural Brain Research, 197(1), 205-209. doi:https://doi.org/ 10.1016/j.bbr.2008.08.037

Ahrens, A. M., Nobile, C. W., Page, L. E., Maier, E. Y., Duvauchelle, C. L., \& Schallert, T. (2013). Individual differences in the conditioned and unconditioned rat $50-\mathrm{kHz}$ ultrasonic vocalizations elicited by repeated amphetamine exposure. Psychopharmacology, 229(4), 687-700. doi:https://doi.org/10.1007/s00213-013-3130-9

Armstrong, V., Nazarian, A., Zavala, A. R., Krall, C. M., Crawford, C. A., \& McDougall, S. A. (2001). Effects of acute and repeated methamphetamine treatment on the ultrasonic vocalizations of postnatal rats. Pharmacology Biochemistry and Behavior, 70(2/3), 273-278. doi: https://doi.org/10.1016/S0091-3057(01)00607-4

Avvisati, R., Contu, L., Stendardo, E., Michetti, C., Montanari, C., Scattoni, M. L., \& Badiani, A. (2016). Ultrasonic vocalization in 
rats self-administering heroin and cocaine in different settings: Evidence of substance-specific interactions between drug and setting. Psychopharmacology, 233(8), 1501-1511. doi:https://doi.org/ 10.1007/s00213-016-4247-4

Bardin, L., Gregoire, S., Aliaga, M., Malfetes, N., Vitton, O., Ladure, P., ... Depoortère, R. (2010). Comparison of milnacipran, duloxetine and pregabalin in the formalin pain test and in a model of stressinduced ultrasonic vocalizations in rats. Neuroscience Research, 66(2), 135-140. doi:https://doi.org/10.1016/j.neures.2009.10.009

Barker, D. J., Herrera, C., \& West, M. O. (2014). Automated detection of $50-\mathrm{kHz}$ ultrasonic vocalizations using template matching in XBAT. Journal of Neuroscience Methods, 236, 68-75. doi:https://doi.org/ 10.1016/j.jneumeth.2014.08.007

Barker, D. J., \& Johnson, A. M. (2017). Automated acoustic analysis of $50-\mathrm{kHz}$ ultrasonic vocalizations using template matching and contour analysis. The Journal of the Acoustical Society of America, 141(3), EL281-EL286. doi:https://doi.org/10.1121/1.4977990

Basken, J. N., Connor, N. P., \& Ciucci, M. R. (2012). Effect of aging on ultrasonic vocalizations and laryngeal sensorimotor neurons in rats. Experimental Brain Research, 219(3), 351-361. doi:https://doi.org/ 10.1007/s00221-012-3096-6

Bell, R. L., Hauser, S., Rodd, Z. A., Liang, T., Sari, Y., McClintick, J., ... Engleman, E. A. (2016). A genetic animal model of alcoholism for screening medications to treat addiction. In R. L. Bell \& S. Rahman (Eds.), International review of neurobiology (Vol. 126, pp. 179261). Amsterdam, The Netherlands: Elsevier. doi:https://doi.org/ 10.1016/bs.irn.2016.02.017

Bell, R. L., Sable, H. J. K., Colombo, G., Hyytia, P., Rodd, Z. A., \& Lumeng, L. (2012). Animal models for medications development targeting alcohol abuse using selectively bred rat lines: Neurobiological and pharmacological validity. Pharmacology Biochemistry and Behavior, 103(1), 119-155. doi:https://doi.org/ 10.1016/j.pbb.2012.07.007

Berger, A. L., Williams, A. M., McGinnis, M. M., \& Walker, B. M. (2013). Affective cue-induced escalation of alcohol selfadministration and increased $22-\mathrm{kHz}$ ultrasonic vocalizations during alcohol withdrawal: Role of kappa-opioid receptors. Neuropsychopharmacology: Official Publication of the American College of Neuropsychopharmacology, 38(4), 647-654. doi:https:// doi.org/10.1038/npp.2012.229

Brenes, J. C., Lackinger, M., Höglinger, G. U., Schratt, G., Schwarting, R. K. W., \& Wöhr, M. (2016). Differential effects of social and physical environmental enrichment on brain plasticity, cognition, and ultrasonic communication in rats. Journal of Comparative Neurology, 524(8), 1586-1607. doi:https://doi.org/10.1002/cne. 23842

Brudzynski, S. M. (2013). Ethotransmission: Communication of emotional states through ultrasonic vocalization in rats. Current Opinion in Neurobiology, 23(3), 310-317. doi:https://doi.org/10. 1016/j.conb.2013.01.014

Brudzynski, S. M. (2015). Pharmacology of ultrasonic vocalizations in adult rats: Significance, call classification and neural substrate. Current Neuropharmacology, 13(2), 180-192. doi:https://doi.org/ 10.2174/1570159X13999150210141444

Brudzynski, S. M., Bihari, F., Ociepa, D., \& Fu, X. W. (1993). Analysis of $22 \mathrm{kHz}$ ultrasonic vocalization in laboratory rats: Long and short calls. Physiology and Behavior, 54(2), 215-221. doi:https://doi. org/10.1016/0031-9384(93)90102-L

Buck, C. L., Vendruscolo, L. F., Koob, G. F., \& George, O. (2014). Dopamine D1 and $\mu$-opioid receptor antagonism blocks anticipatory $50 \mathrm{kHz}$ ultrasonic vocalizations induced by palatable food cues in Wistar rats. Psychopharmacology, 231(5), 929-937. doi:https://doi. org/10.1007/s00213-013-3307-2

Burgdorf, J., \& Panksepp, J. (2006). The neurobiology of positive emotions. Neuroscience and Biobehavioral Reviews, 30(2), 173-187. doi:https://doi.org/10.1016/j.neubiorev.2005.06.001
Burgdorf, J., Wood, P. L., Kroes, R. A., Moskal, J. R., \& Panksepp, J. (2007). Neurobiology of $50-\mathrm{kHz}$ ultrasonic vocalizations in rats: Electrode mapping, lesion, and pharmacology studies. Behavioural Brain Research, 182(2), 274-283. doi:https://doi.org/10.1016/j.bbr. 2007.03.010

Chen, X., Li, Y., Li, S., \& Kirouac, G. J. (2012). Early fear as a predictor of avoidance in a rat model of post-traumatic stress disorder. Behavioural Brain Research, 226(1), 112-117. doi:https://doi.org/ 10.1016/j.bbr.2011.09.004

Ciucci, M. R., Ahrens, A. M., Ma, S. T., Kane, J. R., Windham, E. B., Woodlee, M. T., \& Schallert, T. (2009). Reduction of dopamine synaptic activity: Degradation of $50-\mathrm{kHz}$ ultrasonic vocalization in rats. Behavioral Neuroscience, 123(2), 328-336. doi:https://doi.org/ 10.1037/a0014593

Crabbe, J. C., Phillips, T. J., Kosobud, A., \& Belknap, J. K. (1990). Estimation of genetic correlation: Interpretation of experiments using selectively bred and inbred animals. Alcoholism: Clinical and Experimental Research, 14(2), 141-151. doi:https://doi.org/ 10.1111/j.1530-0277.1990.tb00461.x

Engleman, E. A., McBride, W. J., Li, T.-K., Lumeng, L., \& Murphy, J. M. (2003). Ethanol drinking experience attenuates (-)sulpiride-induced increases in extracellular dopamine levels in the nucleus accumbens of alcohol-preferring (P) rats. Alcoholism: Clinical and Experimental Research, 27(3), 424-431. doi:https://doi.org/10. 1097/01.ALC.0000056618.57931.A5

Engleman, E. A., McBride, W. J., Wilber, A. A., Shaikh, S. R., Eha, R. D., Lumeng, L., ... Murphy, J. M. (2000). Reverse microdialysis of a dopamine uptake inhibitor in the nucleus accumbens of alcoholpreferring rats: Effects on dialysate dopamine levels and ethanol intake. Alcoholism: Clinical and Experimental Research, 24(6), 795-801. doi:https://doi.org/10.1111/j.1530-0277.2000.tb02058.x

Enoch, M. A., \& Goldman, D. (2001). The genetics of alcoholism and alcohol abuse. Current Psychiatry Reports, 3(2), 144-151. doi: https://doi.org/10.1007/s11920-001-0012-3

Furlanetti, L. L., Coenen, V. A., Aranda, I. A., \& Döbrössy, M. D. (2015). Chronic deep brain stimulation of the medial forebrain bundle reverses depressive-like behavior in a hemiparkinsonian rodent model. Experimental Brain Research, 233(11), 3073-3085. doi:https:// doi.org/10.1007/s00221-015-4375-9

Gongwer, M. A., Murphy, J. M., McBride, W. J., Lumeng, L., \& Li, T. K. (1989). Regional brain contents of serotonin, dopamine and their metabolites in the selectively bred high- and low-alcohol drinking lines of rats. Alcohol, 6(4), 317-320. doi:https://doi.org/10.1016/ 0741-8329(89)90089-X

Johnson, A. M., Grant, L. M., Schallert, T., \& Ciucci, M. R. (2015). Changes in rat $50-\mathrm{kHz}$ ultrasonic vocalizations during dopamine denervation and aging: Relevance to neurodegeneration. Current Neuropharmacology, 13(2), 211-219. doi:https://doi.org/10.2174/ 1570159X1302150525122416

Kaniuga, E., Taracha, E., Stepień, T., Wierzba-Bobrowicz, T., Płaźnik, A., \& Chrapusta, S. J. (2016). Rats showing low and high sensitization of frequency-modulated $50-\mathrm{kHz}$ vocalization response to amphetamine differ in amphetamine-induced brain Fos expression. Brain Research, 1648(Pt. A), 356-364. doi:https://doi.org/10.1016/j. brainres.2016.08.008

Kearns, M., \& Ron, D. (1999). Algorithmic stability and sanity-check bounds for leave-one-out cross-validation. Neural Computation, 11(6), 1427-1453. doi:https://doi.org/10.1162/ 089976699300016304

Knutson, B., Burgdorf, J., \& Panksepp, J. (2002). Ultrasonic vocalizations as indices of affective states in rats. Psychological Bulletin, 128(6), 961-977. doi:https://doi.org/10.1037//0033-2909.128.6.961

Lindquist, D. H., Jarrard, L. E., \& Brown, T. H. (2004). Perirhinal cortex supports delay fear conditioning to rat ultrasonic social signals. The Journal of Neuroscience: The Official Journal of the Society for 
Neuroscience, 24(14), 3610-3617. doi:https://doi.org/10.1523/ JNEUROSCI.4839-03.2004

Ma, S. T., Maier, E. Y., Ahrens, A. M., Schallert, T., \& Duvauchelle, C. L. (2010). Repeated intravenous cocaine experience: Development and escalation of pre-drug anticipatory $50-\mathrm{kHz}$ ultrasonic vocalizations in rats. Behavioural Brain Research, 212(1), 109-114. doi:https:// doi.org/10.1016/j.bbr.2010.04.001

Machold, R. P. (2013). Loss of rostral brainstem cholinergic activity results in decreased ultrasonic vocalization behavior and altered sensorimotor gating. Behavioural Brain Research, 256, 51-55. doi:https://doi.org/10.1016/j.bbr.2013.06.030

Maier, E. Y., Abdalla, M., Ahrens, A. M., Schallert, T., \& Duvauchelle, C. L. (2012). The missing variable: Ultrasonic vocalizations reveal hidden sensitization and tolerance-like effects during long-term cocaine administration. Psychopharmacology, 219(4), 1141-1152. doi: https://doi.org/10.1007/s00213-011-2445-7

McBride, W. J., Chernet, E., Dyr, W., Lumeng, L., \& Li, T. K. (1993). Densities of dopamine D2 receptors are reduced in CNS regions of alcohol-preferring P rats. Alcohol, 10(5), 387-390. doi:https://doi. org/10.1016/0741-8329(93)90025-J

McBride, W. J., Kimpel, M. W., McClintick, J. N., Ding, Z. M., Hauser, S. R., Edenberg, H. J., ... Rodd, Z. A. (2013a). Changes in gene expression within the ventral tegmental area following repeated excessive binge-like alcohol drinking by alcohol-preferring $(\mathrm{P})$ rats. Alcohol, 47(5), 367-380. doi:https://doi.org/10.1016/j.alcohol. 2013.04.002

McBride, W. J., Kimpel, M. W., McClintick, J. N., Ding, Z. M., Hyytia, P., Colombo, G., ... Bell, R. L. (2013b). Gene expression within the extended amygdala of 5 pairs of rat lines selectively bred for high or low ethanol consumption. Alcohol, 47(7), 517-529. doi:https://doi. org/10.1016/j.alcohol.2013.08.004

McBride, W. J., Rodd, Z. A., Bell, R. L., Lumeng, L., \& Li, T.-K. (2014). The alcohol-preferring $(\mathrm{P})$ and high-alcohol-drinking (HAD) ratsAnimal models of slcoholism. Alcohol (Fayetteville, N.Y.), 48(3), 209-215. doi:https://doi.org/10.1016/j.alcohol.2013.09.044

Mittal, N., Thakore, N., Reno, J. M., Bell, R. L., Maddox, W. T., Schallert, T., \& Duvauchelle, C. L. (2017). Alcohol-naïve USVs distinguish male HAD-1 from LAD-1 rat strains. Alcohol. doi: https://doi.org/10.1016/j.alcohol.2017.09.003

Morzorati, S. L. (1998). VTA dopamine neuron activity distinguishes alcohol-preferring (P) rats from Wistar rats. Alcoholism: Clinical and Experimental Research, 22(4), 854-857. doi:https://doi.org/ 10.1111/j.1530-0277.1998.tb03879.x

Morzorati, S. L., \& Marunde, R. L. (2006). Comparison of VTA dopamine neuron activity in lines of rats selectively bred to prefer or avoid alcohol. Alcoholism: Clinical and Experimental Research, 30(6), 991-997. doi:https://doi.org/10.1111/j.1530-0277.2006. 00113.x

Murphy, J. M., McBride, W. J., Lumeng, L., \& Li, T. K. (1982). Regional brain levels of monoamines in alcohol-preferring and -nonpreferring lines of rats. Pharmacology, Biochemistry and Behavior, 16(1), 145-149. doi:https://doi.org/10.1016/0091-3057(82)90026-0

Murphy, J. M., McBride, W. J., Lumeng, L., \& Li, T. K. (1987). Contents of monoamines in forebrain regions of alcohol-preferring $(\mathrm{P})$ and nonpreferring (NP) lines of rats. Pharmacology, Biochemistry and Behavior, 26(2), 389-392. doi:https://doi.org/10.1016/00913057(87)90134-1

Murphy, J. M., Stewart, R. B., Bell, R. L., Badia-Elder, N. E., Carr, L. G., McBride, W. J., ... Li, T. K. (2002). Phenotypic and genotypic characterization of the Indiana university rat lines selectively bred for high and low alcohol preference. Behavior Genetics, 32(5), 363 388. doi:https://doi.org/10.1023/A:1020266306135

Pan, J., Palmateer, J., Schallert, T., Hart, M., Pandya, A., Vandenbark, A. A., ... Hurn, P. D. (2014). Novel humanized recombinant T cell receptor ligands protect the female brain after experimental stroke.
Translational Stroke Research, 5(5), 577-585. doi:https://doi.org/ 10.1007/s12975-014-0345-y

Parsana, A. J., Moran, E. E., \& Brown, T. H. (2012). Rats learn to freeze to $22-\mathrm{kHz}$ ultrasonic vocalizations through autoconditioning. Behavioural Brain Research, 232(2), 395-399. doi:https://doi.org/ 10.1016/j.bbr.2012.03.031

Popik, P., Kos, T., Pluta, H., Nikiforuk, A., Rojek, K., \& Ryguła, R. (2014). Inhibition of the glucocorticoid synthesis reverses stressinduced decrease in rat's $50-\mathrm{kHz}$ ultrasonic vocalizations. Behavioural Brain Research, 260, 53-57. doi:https://doi.org/10. 1016/j.bbr.2013.11.029

Portfors, C. V. (2007). Types and functions of ultrasonic vocalizations in laboratory rats and mice. Journal of the American Association for Laboratory Animal Science: JAALAS, 46(1), 28-34. Retrieved from http://www.ncbi.nlm.nih.gov/pubmed/17203913

Reno, J. M., \& Duvauchelle, C. L. (2014). Response to: Making WAAVES in the vocalization community: How big is the splash? Journal of Neuroscience Methods, 221, 230-232. doi:https://doi. org/10.1016/j.jneumeth.2013.09.008

Reno, J. M., Marker, B., Cormack, L. K., Schallert, T., \& Duvauchelle, C. L. (2013). Automating ultrasonic vocalization analyses: The WAAVES program. Journal of Neuroscience Methods, 219(1), 155-161. doi:https://doi.org/10.1016/j.jneumeth.2013.06.006

Reno, J. M., Thakore, N., Cormack, L. K., Schallert, T., Bell, R. L., Maddox, W. T., \& Duvauchelle, C. L. (2017). Negative affectassociated USV acoustic characteristics predict future excessive alcohol drinking and alcohol avoidance in male $\mathrm{P}$ and NP rats. Alcoholism: Clinical and Experimental Research, 1-12. doi: https://doi.org/10.1111/acer.13344

Reno, J. M., Thakore, N., Gonzales, R., Schallert, T., Bell, R. L., Maddox, W. T., \& Duvauchelle, C. L. (2015). Alcohol-preferring P rats emit spontaneous $22-28 \mathrm{khz}$ ultrasonic vocalizations that are altered by acute and chronic alcohol experience. Alcoholism: Clinical and Experimental Research, 39(5), 843-852. doi:https://doi.org/10. 1111/acer.12706

Rezvani, A. H., Slade, S., Wells, C., Petro, A., Lumeng, L., Li, T.-K., ... Levin, E. D. (2010). Effects of sazetidine-A, a selective alpha4beta2 nicotinic acetylcholine receptor desensitizing agent on alcohol and nicotine self-administration in selectively bred alcohol-preferring (P) rats. Psychopharmacology, 211(2), 161-174. doi:https://doi. org/10.1007/s00213-010-1878-8

Roccaro-Waldmeyer, D. M., Babalian, A., Müller, A., \& Celio, M. R. (2016). Reduction in $50-\mathrm{kHz}$ call-numbers and suppression of tickling-associated positive affective behaviour after lesioning of the lateral hypothalamic parvafox nucleus in rats. Behavioural Brain Research, 298(Pt. B), 167-180. doi:https://doi.org/10.1016/j. bbr.2015.11.004

Rodd, Z. A., Bell, R. L., Sable, H. J. K., Murphy, J. M., \& McBride, W. J. (2004). Recent advances in animal models of alcohol craving and relapse. Pharmacology Biochemistry and Behavior, 79(3), 439-450. doi:https://doi.org/10.1016/j.pbb.2004.08.018

Sacks, J. J., Gonzales, K. R., Bouchery, E. E., Tomedi, L. E., \& Brewer, R. D. (2015). 2010 national and state costs of excessive alcohol consumption. American Journal of Preventive Medicine, 49(5), 73-79. doi:https://doi.org/10.1016/j.amepre.2015.05.031

Sahr, A. E., Thielen, R. J., Lumeng, L., Li, T.-K., \& McBride, W. J. (2004). Long-lasting alterations of the mesolimbic dopamine system after periadolescent ethanol drinking by alcohol-preferring rats. Alcoholism: Clinical and Experimental Research, 28(5), 702-11. doi:https://doi.org/10.1097/01.ALC.0000125344.79677.1C

Scattoni, M. L., Crawley, J., \& Ricceri, L. (2009). Ultrasonic vocalizations: A tool for behavioural phenotyping of mouse models of neurodevelopmental disorders. Neuroscience and Biobehavioral Reviews, 33(4), 508-515. doi:https://doi.org/10.1016/j.neubiorev. 2008.08.003 
Srisontiyakul, J., Kastman, H. E., Krstew, E. V., Govitrapong, P., \& Lawrence, A. J. (2016). The nicotinic $\alpha 6$-subunit selective antagonist bPiDI reduces alcohol self-administration in alcohol-preferring rats. Neurochemical Research, 41(12), 3206-3214. doi:https://doi. org/10.1007/s11064-016-2045-3

Steele, F. F., Whitehouse, S. C., Aday, J. S., \& Prus, A. J. (2017). Neurotensin NTS1 and NTS2 receptor agonists produce anxiolytic-like effects in the $22-\mathrm{kHz}$ ultrasonic vocalization model in rats. Brain Research, 1658, 31-35. doi:https://doi.org/10.1016/j. brainres.2017.01.012

Stewart, R. B., \& Li, T. K. (1997). The neurobiology of alcoholism in genetically selected rat models. Alcohol Health and Research World, 21(2), 169-176. Retrieved from http://www.ncbi.nlm.nih.gov/ pubmed/15704355

Tawa, E. A., Hall, S. D., \& Lohoff, F. W. (2016). Overview of the genetics of alcohol use disorder. Alcohol and Alcoholism, 51(5), 507-514. doi:https://doi.org/10.1093/alcalc/agw046

Thakore, N., Reno, J. M., Gonzales, R. A., Schallert, T., Bell, R. L., Maddox, W. T., \& Duvauchelle, C. L. (2016). Alcohol enhances unprovoked 22-28 kHz USVs and suppresses USV mean frequency in high alcohol drinking (HAD-1) male rats. Behavioural Brain Research, 302, 228-236. doi:https://doi.org/10.1016/j.bbr.2016.01. 042

Thielen, R. J., Engleman, E. A., Rodd, Z. A., Murphy, J. M., Lumeng, L., Li, T.-K., \& McBride, W. J. (2004). Ethanol drinking and deprivation alter dopaminergic and serotonergic function in the nucleus accumbens of alcohol-preferring rats. The Journal of Pharmacology and Experimental Therapeutics, 309(1), 216-225. doi:https://doi.org/10.1124/jpet.103.059790

Tizabi, Y., Getachew, B., Davila-Garcia, M., \& Taylor, R. E. (2001). Alcohol preference: Association with reduced striatal nicotinic receptors. Alcohol and Alcoholism (Oxford, Oxfordshire), 36(4), 318322. Retrieved from http://www.ncbi.nlm.nih.gov/pubmed/ 11468132
Venables, W. N., \& Ripley, B. D. (2002). Modern applied statistics with S. Technometrics, 45(1). doi:https://doi.org/10.1198/tech.2003.s33

Wang, H., Liang, S., Burgdorf, J., Wess, J., \& Yeomans, J. (2008). Ultrasonic vocalizations induced by sex and amphetamine in M2, M4, M5 muscarinic and D2 dopamine receptor knockout mice. PLOS ONE, 3(4), 30-33. doi:https://doi.org/10.1371/journal.pone. 0001893

Weisbroth, S. H. (1969). The origin of the Long-Evans rat and a review of the inheritance of coat colors in rats (Rattus norvegicus). Laboratory Animal Care, 19(5), 733-737. Retrieved from http://www.ncbi.nlm. nih.gov/pubmed/4247437

Weiss, F., Lorang, M. T., Bloom, F. E., \& Koob, G. F. (1993). Oral alcohol self-administration stimulates dopamine release in the rat nucleus accumbens: Genetic and motivational determinants. The Journal of Pharmacology and Experimental Therapeutics, 267(1), 250258. Retrieved from http://www.ncbi.nlm.nih.gov/pubmed/ 8229752

Williams, S. N., \& Undieh, A. S. (2016). Dopamine-sensitive signaling mediators modulate psychostimulant-induced ultrasonic vocalization behavior in rats. Behavioural Brain Research, 296, 1-6. doi: https://doi.org/10.1016/j.bbr.2015.08.008

Wöhr, M., Houx, B., Schwarting, R. K. W., \& Spruijt, B. (2008). Effects of experience and context on $50-\mathrm{kHz}$ vocalizations in rats. Physiology and Behavior, 93(4/5), 766-776. doi:https://doi.org/10. 1016/j.physbeh.2007.11.031

Wöhr, M., \& Schwarting, R. K. W. (2013). Affective communication in rodents: Ultrasonic vocalizations as a tool for research on emotion and motivation. Cell and Tissue Research, 354(1), 81-97. doi: https://doi.org/10.1007/s00441-013-1607-9

Zhou, F. C., Zhang, J. K., Lumeng, L., \& Li, T. K. (1995). Mesolimbic dopamine system in alcohol-preferring rats. Alcohol, 12(5), 403412. doi:https://doi.org/10.1016/0741-8329(95)00010-O 